This provisional PDF corresponds to the article as it appeared upon acceptance. A copyedited and fully formatted version will be made available soon. The final version may contain major or minor changes.

\title{
The functional manual therapy intervention in infants with non-synostotic plagiocephaly: A pilot study
}

\author{
MARIANGELA BILLI, ANGELO GRECO, PAOLA COLONNELLI, GIORDANA VOLPI, \\ DONATELLA VALENTE, GIOVNNI GALEOTO
}

Minerva Pediatrica 2017 Oct 25

DOI: 10.23736/S0026-4946.17.04838-1

Article type: Original Article

(C) 2017 EDIZIONI MINERVA MEDICA

Article first published online: October 25, 2017

Manuscript accepted: September 20, 2017

Manuscript received: October 26, 2016

Subscription: Information about subscribing to Minerva Medica journals is online at: http://www.minervamedica.it/en/how-to-order-journals.php

Reprints and permissions: For information about reprints and permissions send an email to: journals.dept@minervamedica.it - journals2.dept@minervamedica.it - journals6.dept@minervamedica.it 
The functional manual therapy intervention in infants with nonsynostotic plagiocephaly: A pilot study

\author{
Mariangela Billi ${ }^{1}$, Angelo Greco ${ }^{2}$, Paola Colonneli ${ }^{2}$, Giordana Volpi ${ }^{2}$, Donatella \\ Valente ${ }^{1}$, Giovanni Galeoto ${ }^{2 *}$
}

${ }^{1}$ Department of Paediatrics and Child Neuropsychiatry, "Sapienza" University of Rome, Italy; ${ }^{2}$ Department of Anatomical, Histological, Forensic and Orthopaedic Sciences, "Sapienza" University of Rome, Italy

Conflicts of interest: The authors declare that there is no conflict of interests regarding the publication of this paper.

\title{
Acknowledgements
}

We thank all the participants who agreed to join this study.

"Corresponding Author:

Galeoto Giovanni, Piazzale Aldo Moro 5, 00185 Rome

mail:usifi.aifilazio@gmail.com 
Abstract

BACKGROUND: To document the evolution of cranial asymmetries in infants with signs of nonsynostotic occipital plagiocephaly (NSOP) who were undergo to many functional manual therapy treatments (in addition to the standard positioning recommendations) as well as to determine the feasibility of this methodology to conduct an outcome research investigating the impact of this intervention for infants with NSOP.

METHODS: Pilot clinical standardization project using pre-post design in which 10 infants participated. Nine infants presented an initial Oblique Diameter Difference Index (ODDI) over 104\%, three an initial Ear Deviation Index (EDI) over 4\%, and three a Cranial Proportional Index (CPI) over 90\%.

Interventions: Infants received three functional manual therapy treatments for week during the first month of intervention and two ones for week during the second month.

RESULTS: Plagiocephalometric measurememts were administered at the first assessment pre-intervention (T0), after 30 days (+/-5) (T1) and at a third time after 60 days $(+/ 5)$ of treatment (T2).

Results: 9/10 participants showed a significant decrease in ODDI under $104 \%$ between T0 and T2 assessments. 5/10 infants showed an EDI under 4\%, and 3/10 showed a value about $0 \%$. 3/10 maintained their CPI over $90 \%$ with a considerable decrease. 
CONCLUSIONS: These clinical findings support the hypothesis that functional manual therapy treatments contribute to the improvement of cranial asymmetries in infants younger than 6.5 months old presenting with NSOP. Key Word: Plagiocephaly, Rehabilitation, therapy 


\section{Introduction}

Plagiocephaly is usually described as a cranial deformity involving primarily a unilateral flattening of the occiput. Literature indicates two types of plagiocephaly: (1) synostotic plagiocephaly (malformation due to premature fusion of the cranial sutures) and (2) nonsynostotic occipital plagiocephaly (NSOP).

NSOP is reported to be caused by mechanical strains such as traction or compression applied before, during or after birth starting progressively by an occipital flattening to the rest of cranial vault.

Since the "Back to sleep" campaign initiated by the American Academy of Pediatrics (AAP) in 1992 to reduce the incidence of Sudden Infant Death Syndrome, this type of skull deformity has become more prevalent and could be observed in $19.7 \%$ of healthy newborns [2].

A number of observational studies suggest that plagiocephaly may be related to delays and impairments in various aspects of an infant's functioning.[3-4]

Available findings show that children with this condition are more prone to developing problems related to: postural compensations [5-6], musculo-skeletal dysfunction, visual perception and opthalmic dysfunction [7-8], temporomandibular articulation [9] and developmental achievements. [10-11]

NSOP is generally defined as including the presence of an Oblique Diameter Difference Index (ODDI) of $104 \%$ or more[12-13].

ODDI is calculated by the ratio within the difference beetween the oblique diameter left (ODL) and the oblique diameter right (ODR). ODL and ODR lines are drawn from points located $40^{\circ}$ either side of the anteroposterior (AP) line drawned by the nose and the middle occipital point. The angle of $40^{\circ}$ is chosen because this has been used by other authors formerly, probably because the differences 
between the diameters of the typical shape of the skull at these angles are the most outstanding.

The ratio between the ODL and the ODR is calculated as the longest/shortest diameter/100 and is called oblique diameter difference index (ODDI). [14]

Despite popular belief, research findings suggest that without intervention, NSOP, does not resolve in time in the majority of the cases. [15-17]

Interventions for NSOP usually include: counter-positioning indicated for mild cases of plagiocephaly [18], physical therapy added to counterpositioning when torticollis is associated with the presence of skull deformity [17], and cranial orthosis, particularly for moderate to severe cases of NSOP or when counterpositioning has failed to correct the asymmetries $[5,19$,$] .$

In our study was considered an early intervention of functional manual therapy added to traditional recommendations for counter-positioning. Earlier intervention has been shown to increase the potential for NSOP correction. $[6,17,19,21,22,23]$.

Through the feasibility and non-invasivity of functional manual therapy intervention is possible intercepting cranial deformities since their primordial manifestations, preventing establishment of severe case of plagiocephaly as in cases of Argenta 3 and 4.

The aim of this study was to document the improvements of cranial asymmetries in an objective manner by ODDI's assessments in infants with signs of NSOP who were to undergo a course of functional manual therapy treatments (in addition to the standard positioning recommendations).

\section{Methods}

\section{Participants}

This document is protected by international copynight laws. No additional reproduction is authorized. It is permitted for personal use to download and save only one file and print only one copy of this Article. It is not permitted to make additional copies (either sporadically or systematically, either printed or electronic) of the Article for any purpose. It is not permitted to distribute the electronic copy of the article through online internet and/or intranet file sharing systems, electronic mailing or any other means which may allow access to the Article. The use of all or any part of the Article for any Commercial Use is not permitted. The creation of derivative works from the Article is not permitted. The production of reprints for personal or commercial use is not frame or use framing techniques to enclose any trademark, logo, or other proprietary information of the Publisher.
fration 
Ten infants with signs of NSOP participated in this analysis have been assessed in Orthopaedic Clinic of Policlinico Umberto I of Rome in the period included since November 2015 until June 2016.

Infants evaluated and treated in the study were either referred by pediatricians. Inclusion criteria for the infants were: to be younger than 6.5 months at the time of the first evaluation, to have a diagnosis or present signs of NSOP (as per recognized definition) and to be at term corrected age if born prematurely. Infants were excluded if there was a documented craniosynostosis, an ongoing cranial orthosis treatment and if presenting with any medical condition. All the patients' parents were asked to sign the informed consent to treatment[24,25].

\section{Measures}

All measures used for this clinical standardization study were those used as part of standard care for infants presenting with NSOP in our institution. At the initial assessment, a routine detailed history was taken including obstetrical, perinatal and postnatal information. In the context of standard assessment for this clientele, tests administered to the infants included plagiocephalometric measurements.

Clinical adaptation of plagiocephalometric measurements, to document the evolution of the oblique diameter left (ODL) and oblique diameter right (ODR), were taken via cranial circumference molds using a low-temperature thermoplastic material (sansplint), as described by Van Vlimmeren et al.[12-14] 
This is a non-invasive, reliable, easy to apply method to assess skull asymmetries. From the ODL and ODR measurements, an Oblique Diameter Difference Index was derived and was used for analyses (table 1).

Furthermore, other parameters were used to reveal ear's asymmetry by the ear deviation index (EDI) calculated by the ratio between the antero-posterior line (AP) and ear deviation (ED), and transversal shape and proportion of the skull were calculated by the ratio between the sinistra-dextra line (SD) and the anteroposterior line (AP) and is called the cranio proportional index (CPI) [12-14] (figure $1)$.

Finally, a parent questionnaire, collecting information on general health of the child, pregnancy condition of the mother, and perinatal data was administered and parents were also asked to fill a daily log of infant activity.

Measurements were performed at three different times: prior to the intervention (pre-test at T0), after 30 days of treatment (+/- 5 days) (T1) and at the $60^{\text {th }}$ day (+/- 5 days) (T2).

\section{Intervention}

Each infant received three functional manual therapy treatments (of about 30 mins) during the first month and two ones during the second month. Functional manual therapy is conducted by applications of manually guided forces to improve physiological function and homeostasis that has been altered by somatic dysfunction. Standard recognized protocols for identifying problems and determining treatment content were followed as per recommendations from the 
Collège d'Études Ostéopathiques, Montréal, Canada and as suggested by Nicette Sergueef. Advice on counter-positioning and stimulation such as those suggested by the Canadian Pediatric Society [24] were demonstrated by the clinician, practiced by parents and given in writing to optimize time spent in prone during awake hours while supervised as well as to stimulate rotation of the head on the side opposite to the flattening.

\section{Data analysis}

Data from all testing sessions were compiled visually and analyzed for trends. To address the study objectives, measures of he infants at each testing session were compared using one-way ANOVAs with repeated measures on the time factor. Specific differences between each assessment times were analyzed using paired t-tests (T1-T3; T3-T5; T1-T5). SPSS version 21 software was used for analyses.

Results

All 10 infants enrolled in this study had a mean of age 3,7 months, and of these 3 were male and 7 female. They were able to complete the assessments and interventions included in this study, thus confirming the feasibility of using such a methodology to undertake a clinical trial.

When assessed with plagiocephalometric measures, 10 of the participants presented an Oblique Diameter Difference Index (ODDI) $>104 \%$, recognized as clinically significant illustrating obvious clinical asymmetry.[12-14] 
Over the course of 2 months treatments, the ODDI in 4/10 participants decreased below the $104 \%$, and $7 / 10$ showed an improvement of their condition, 2 showed a maintenance of their asymmetry at the same ODDI's value, 1 showed a worsening. The average ODDI's value of the 10 participants evolved from $108.9 \%$ (T0) to $105.7 \%(\mathrm{~T} 2)$.

The ANOVA performed on the ODDI revealed no time effect $(F<2.79 ; p<0.08)$ but there was a tendency towards significance between T0 and T2 values as illustrated by the paired t-test values performed on those times alone $(t<2.35 ; \mathrm{p}$ $<0.03$ ) (figure 2).

At T0, three infants showed an EDI score over 4\%, at T2 five infants showed the same one over the $4 \%$. The average EDI improved from $4,21 \%$ to $3,86 \%$ (figure 3 ).

CPI showed that at T0 two infants had a score over 90\%, and at T2 they improve their condition. The average improvement was about 2,12\% passing by $87,99 \%$ to $85,87 \%$ (figure 4 ).

\section{Discussion}

The main objective of this pilot study was to document the evolution of cranial asymmetries in infants with signs of NSOP who were undergone to a course of many functional manual therapy treatments (in addition to the standard counterpositioning recommendations).

Results obtained from plagiocephalometric measurements revealed a significant improvement in ODDI during the follow-up period. The molds are usually found to be clinically relevant as they reflect the parallelogram shaped head. They are enable to show objectively and easily the evolution of cranial asymmetries to parents and service providers. Vlimmeren et al. defined an ODDI score of $104 \%$ or higher as clinically relevant asymmetry of the skull. [27] 
The observable aspects characterizing NSOP are only part of the problem. The compression forces which deforms the skull are absorbed and transmitted to the body as a functional unit and because of this, the global aspect of functional manual therapy treatment deserves particular attention. The impact of the forces which contributed to deforming the skull is thought to be limited not only to the level of the head or cervical region. The NSOP is a problem of asymmetry and the flattened occiput which transforms the axes and planes of condylar movements in regard to the atlas. These asymmetries of the occiput may create postural compensations in order to ensure horizontal gaze. Asymmetries and immaturity of certain movement's components were also noted in our sample and by correcting the main dysfunctions (in order of primarity) found in each infant, all of them showed improvement of cervical mobility and tolerance in prone position. Recommendations of American Academy of Pediatrics (1996) and Canadian Pediatric Society (2004) to place infants in supine to sleep in order to prevent SIDS are largely implemented. It is the responsibility of all service providers to inform parents of the importance of prone play during awake hours. Once the diagnosis of NSOP is made however, our results suggest that early referral to experts could improve asymmetries and facilitate recovery.

Because of the small sample size and the absence of a control group, no definitive conclusions can be made that the decrease in asymmetry observed in our sample were due to the functional manual therapy treatment alone. However, it is reasonable to say that any improvement superior to that reported with positioning alone, the widely accepted intervention for NSOP, could be attributed, 
at least partially, to the intervention. Indeed, infants in our sample improved their average ODDI score by $3,32 \%$.

Van Vlimmeren et al. (2008) using plagiocephalometry, measured a decreased of the ODDI of $1.5 \%$ in the experimental group after a four month standardized intervention program of pediatric physical therapy (children with positional preference aged between 7 weeks and 6 months, children with congenital muscular torticollis were excluded) in comparison to an increase of $0.2 \%$ in the control group who received care as usual [27]. While comparing the results from the present pilot study to the previous research on the subject it suggests that functional manual treatments could contribute to modifying the cranial asymmetries of infants younger than 6.5 months old presenting with signs of NSOP. A larger sample, using a randomized controlled design is recommended to confirm the results of the present exploratory study. Furthermore, our clinical experience and our understanding of the problem leads us to believe that there is a need for more research of the impact of skull asymmetries on the child's development.

\section{Conclusion}

This study demonstrated statistically and clinically significant improvements in cranial asymmetry of infants with NSOP following two months of functional manual therapy intervention (in addition to the standard positioning recommendations). These results suggest that this methodology could contribute to modifying the cranial asymmetries of infants younger than 6.5 months old presenting with signs of NSOP. 
Through plagiocephalometric measurement was possible objectively assessing changes in plagiocephaly by functional manual therapy treatment. This study successfully utilized clinical procedures that could contribute to the creation of standardized protocol both clinically and with a larger-scale randomized controlled trial.

Furthermore, through this intervention is possible to intercept cases of NSOP prematurely, before a progressive development of severe gravity condition and before other protocols in which is indicated starting the intervention at about 5-6 months of age.[28] 


\section{References}

1 Exploring the impact of osteopathic treatment on cranial asymmetries associated with nonsynostotic plagiocephaly in infants. Lessard, Gagnon, Trottier. Complementary therapies in clinical practice 2011

2 Peitsch WK, Keefer $\mathrm{CH}$, LaBrie RA, et al. Incidence of cranial asymmetry in healthy newborns. Pediatrics 2002;110:e72

3 Miller R, Clarren S. Long term developmental outcomes in patients with deformational plagiocephaly. Pediatrics 2000;105:e26.

4 Moss SD. Nonsurgical, nonorthotic treatment of occipital plagiocephaly: what is the natural history of the misshapen neonatal head? Journal of Neurosurgery 1997;87:667e70.

5 Hylton N. Infants with torticollis: the relationship between asymmetric head and neck positioning and postural development. Physical \& Occupational Therapy in Pediatrics 1997;17:91e117.

6 Biggs M. Diagnosis and management of positional head deformity. American Family Physician 2003;67:1953e6.

7 Siatkowski RM, Fortney AC, Nazir SA, et al. Visual field defects in deformational posterior plagiocephaly. Journal of Aapos: American Association for Pediatric Ophthalmology \& Strabismus 2005;9:274e8.Gupta P, Foster J, Crowe S, Papay F, Luciano M, Traboulsi E. Opthalmologic

8 Findings in Patients with Nonsyndromic Plagiocephaly. Journal of Craniofacial Surgery $2003 ; 14: 529 \mathrm{e} 32$.

9 St. John D, Mulliken J, Kablan L. Anthropometric analysis of mandibular asymmetry in infants with deformational posterior plagiocephaly. Journal of Maxillofacial Surgery 2002;60:873e7.

10 Kordestani RK, Panchal J. Neurodevelopment delays in children with deformational plagiocephaly. Plastic and Reconstructive Surgery 2006;118:808e9. 11 Panchal J, Amirsheybani H, Gurwitch R, et al. Neurodevelopment in children with single-suture craniosynostosis and plagiocephaly without synostosis. Plastic and Reconstructive Surgery 2001;108:1492e8.

12 van Vlimmeren LA, Takken T, van Adrichem LN, et al. Plagiocephalometry: a non-invasive method to quantify asymmetry of the skull; a reliability study. European Journal of Pediatrics 2006;165:149e57. 
13 van Vlimmeren LA, van der Graaf Y, Boere-Boonekamp MM, et al. Risk factors for deformational plagiocephaly at birth and at 7 weeks of age: a prospective cohort study. Pediatrics 2007;119:e408e18.

14 Van Adrichem, van Vlimmeren, Dominika Cadanova, Helders, Engelbert, van Neck, Koning.

Validation of a simple method for measuring cranial deformities (plagiocephalometry). The journal of craniofacial surgery 2008

15 Boere-Boonekamp M, Linden-Kniper LV. Positional prevalence in infants and follow-up after two years. Pediatrics 2001;107:339e43.

16 Kane A, Mitchell L, Craven K. Observations on a recent increase in plagiocephaly without synostosis. Pediatrics 1996;97:877e85.

17 Ripley C, Pomatto J, Beals S, Joganic E, Manwaring K, Moss S. Treatment of positional plagiocephaly with dynamic orthotic cranioplasty. Journal of Craniofacial Surgery 1994;5:150e9.

18 Graham Jr JM, Gomez M, Halberg A, et al. Management of deformational plagiocephaly: repositioning versus orthotic therapy. Journal of Pediatrics 2005;146:258e62.

19 Kelly K, Littlefield T, Pomatto J, Ripley C, Beals S, Joganics E. Importance of early recognition and treatment of deformational plagiocephaly with orthotic cranioplasty. Cleft Palate-Craniofacial Journal 1999; 36:127e30

20 Teichgraeber JF, Ault JK, Baumgartner J, et al. Deformational posterior plagiocephaly: diagnosis and treatment. Cleft Palate e Craniofacial Journal 2002;39: 582e6.

21 Littlefield T, Beals S, Manwaring K, et al. Treatment of craniofacial asymmetry with dynamic orthotic cranioplasty. Journal of Craniofacial Surgery 1998; 9:11e7.

22 Sergueef N, Nelson KE, Glonek T, Sergueef N, Nelson KE, Glonek T. Palpatory diagnosis of plagiocephaly. Complementary Therapies in Clinical Practice 2006;12:101e10.

23 Galeoto G, De Santis R, Marcolini A, Cinelli A, Cecchi R. The informed consent in Occupational Therapy: proposal of forms. Giornale Italiano di Medicina del Lavoro Ed Ergonomia. 2016 Apr 1;38(2):107-15. 
24 Galeoto, G., Mollica, R., Astorino, O., Cecchi, R. (2015). Informed consent in physiotherapy: proposal of a form. Giornale italiano di Medicina del Lavoro ed Ergonomia, 37(4), 245.

25 Zweedjik F, Bekaert W. Posterior plagiocephaly: review of the literature and an osteopathic approach. The Magazine of The Dutch Osteopathic Association 2003;4:3e22.

26 Canadian Paediatric Society. Community Paediatrics Committee: recommendations for safe sleeping environments for infants and children. Paediatrics \& Child Health 2004;9:659e63.

27 van Vlimmeren LA, van der Graaf Y, Boere-Boonekamp MM, et al. Effect of pediatric physical therapy on deformational plagiocephaly in children with positional preference: a randomized controlled trial. Archives of Pediatrics \& Adolescent Medicine 2008;162:712e8.

28 Kluba, Kraut, Reinert, Krimmel. What is the optimal time to start helmet therapy in positional plagiocephaly? Plastic and reconstructive surgery. 2011 
Table 1 The reference scores

\begin{tabular}{|l|l|l|l|}
\hline & ODDI $\%$ & CPI $\%$ & EDI $\%$ \\
\hline Normal & $<104$ & $<90$ & $<4$ \\
\hline Light & $104<\mathrm{x}<108$ & $90<\mathrm{x}<95$ & $4<\mathrm{x}<7$ \\
\hline Severe & $108<\mathrm{x}<112$ & $95<\mathrm{x}<100$ & $7<\mathrm{x}<10$ \\
\hline $\begin{array}{l}\text { Much } \\
\text { severe }\end{array}$ & $>112$ & $>100$ & $>10$ \\
\hline
\end{tabular}



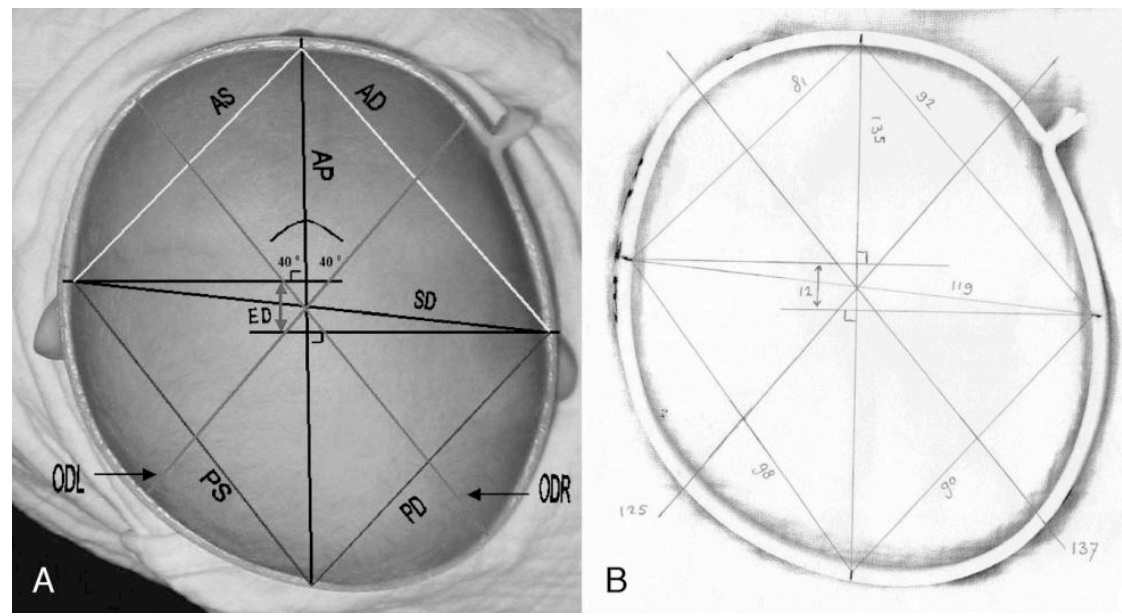

Figure 1 Illustration of plagiocephalometry: Asymmetric skull in positional plagiocephaly of right occiput (4-month-old male): A) Photograph of the child with the thermoplastic ring and landmarks. The digitally drawn lines are made to illustrate the agreement with the paper copy and to explain the names of the lines. B) Paper copy of the same ring with drawn and measured lines. AP indicates anterior-posterior; SD,sinistra-dextra; ED, ear deviation; ODL, oblique diameter left; ODR, oblique diameter right; ODD, ODL minus ODR

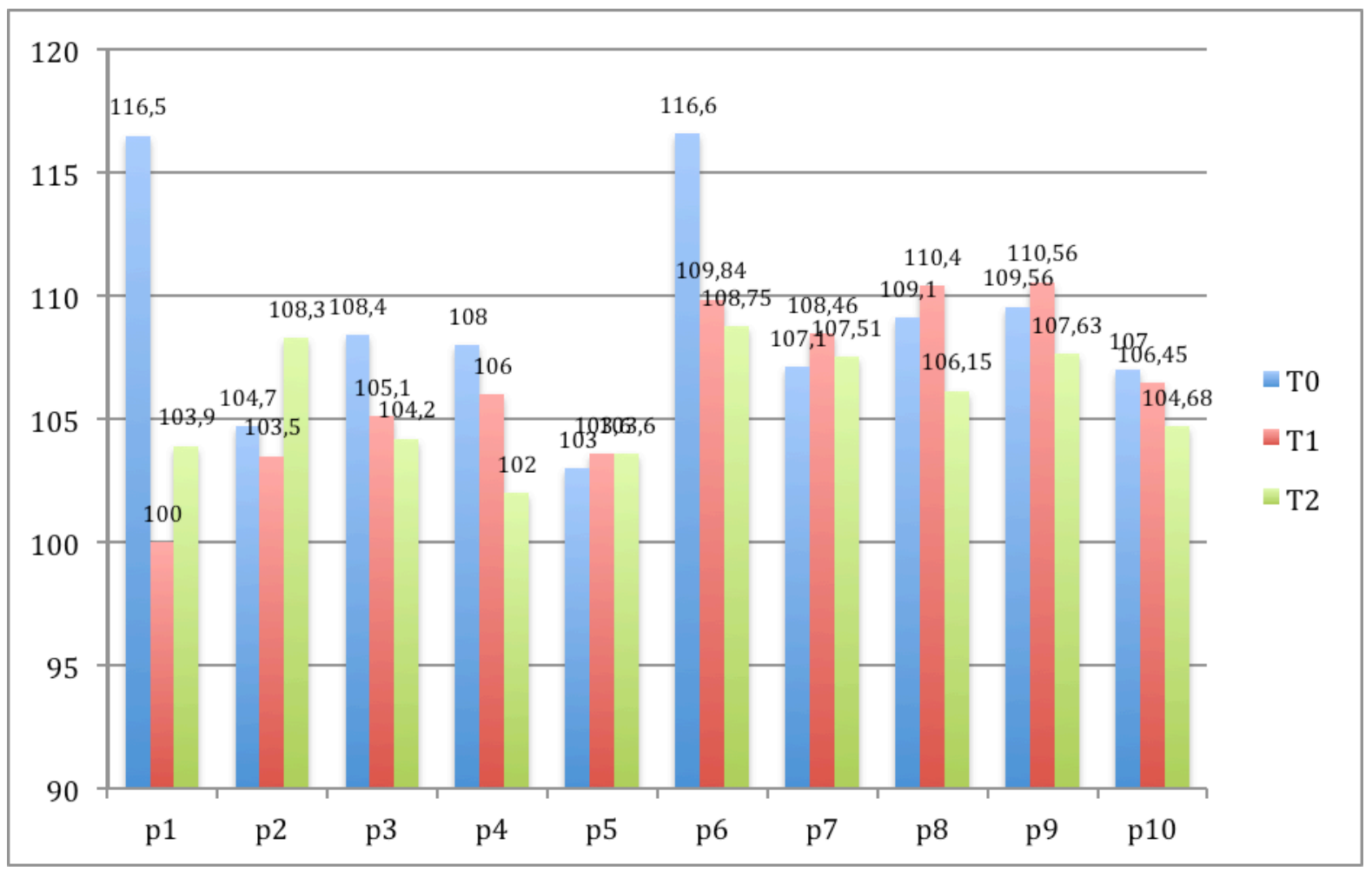

Figure 2 ODDI score

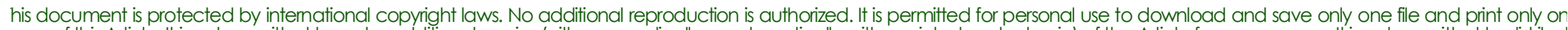

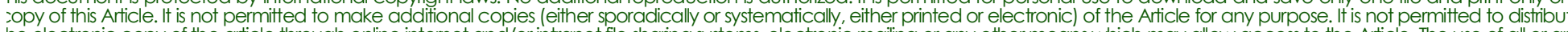

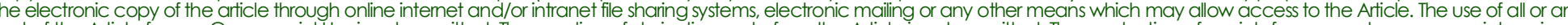




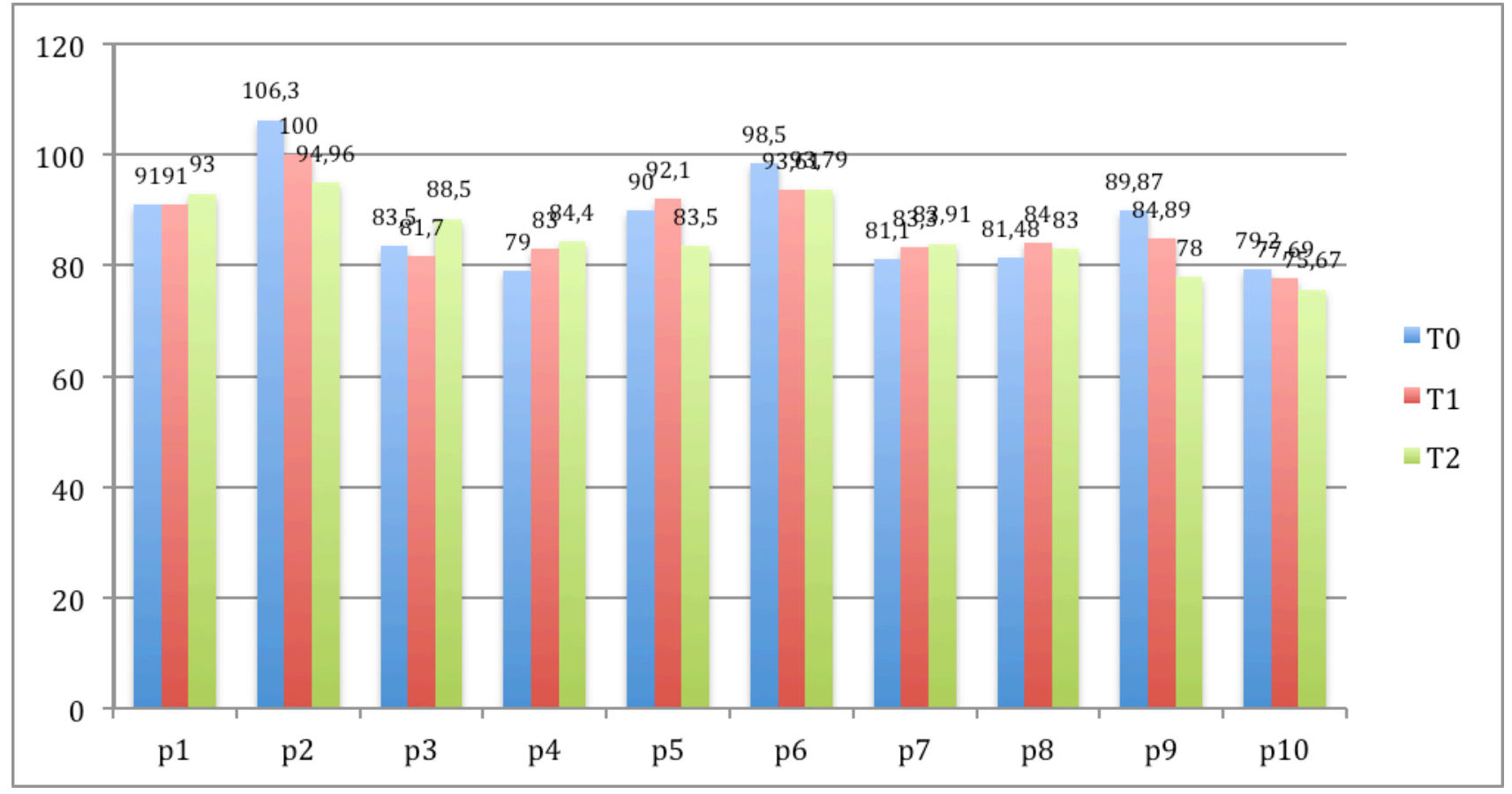

Figura 3 EDI score



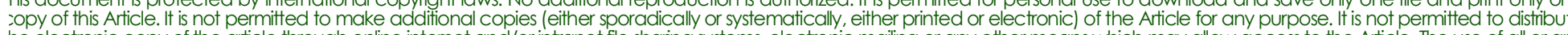

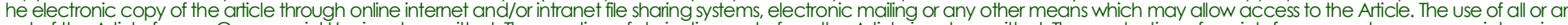




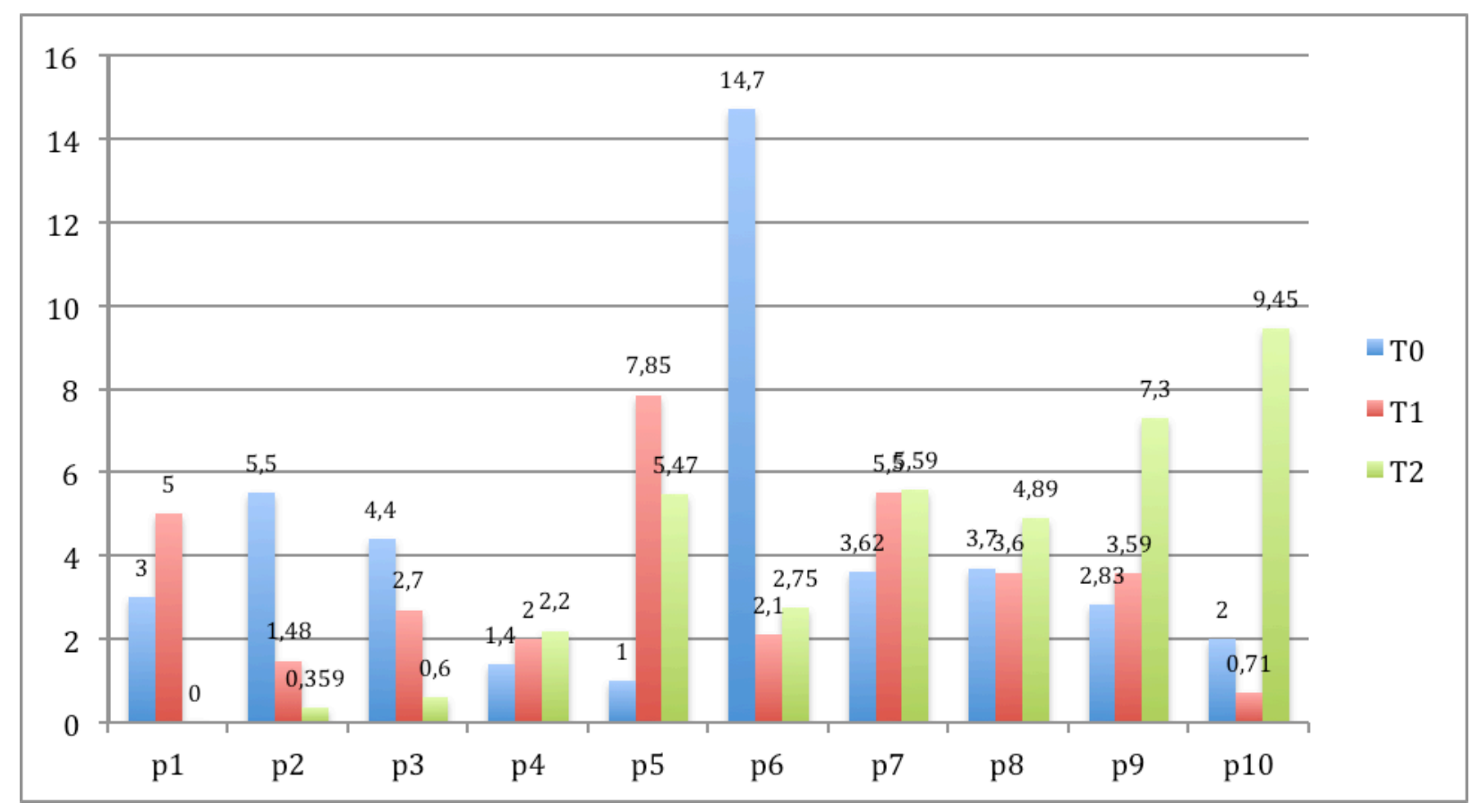

Figure 1 CPI score

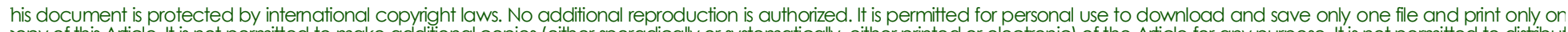

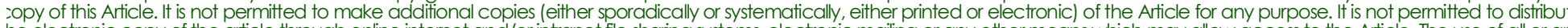

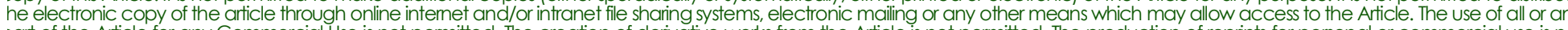

